\title{
Funciones y urgencias de la Universidad
}

\author{
Aura Elena Bernal de Rojas. \\ aurebes@hotmail.com \\ Universidad y Compromiso Nacional \\ Universidad Surcolombiana.
}

\section{Funciones de la Universidad}

as funciones son las actividades, ejercicios o gestiones que la institución desempeña o hace.

El quehacer de la 'universitas', afianzada en sus notas distintivas (universal, cientifica, corporativa y autónoma), tuvo como meta al hombre, a la ciencia y a la sociedad, así, sus funciones de investigación, docencia y extensión. Docencia, en la formación de la persona y su preparación para las ciencias, las artes y las profesiones; investigación, en la búsqueda, sistematización y producción del conocimiento, medio de formación y herramienta para la solución de problemas científicos y sociales; y Extensión, en la socialización del conocimiento, aporte a la comunidades en las diversas actividades y difusión de los valores de la cultura en procura del mejoramiento de las condiciones de vida de la población.

Las funciones de la universidad adquirieron sentido en la medida en que sus actividades estuvieron orientadas a dar respuestas a las necesidades de la sociedad, no para imitar, sino para crear y contribuir al diseño de un futuro nuevo y a la formación de un ser humano universal. La universidad debió estar en la vanguardia de la búsqueda de la verdad para contribuir al difícil tránsito de la humanidad hacia el futuro.

\section{La Docencia Universitaria}

La Universidad nace con la docencia, legado de las antiguas escuelas y academias del mundo de occidente, agremiadas y constituidas en la universitas con identidad en sus notas 0 rasgos distintivos en función del conocimiento.

El contenido de la docencia en el momento del surgimiento de la universidad lo fue el del conocimiento existente en la época: las artes liberales (siete artes: Trivium o artes de la palabra- Gramática, Dialéctica y Retórica; y el Quadrivium o artes de las disciplinas, de las cosas y las cantidades-Aritmética, Geometría, Astronomía y Música), las profesiones, organizadas en las Facultas (Teología, Medicina y Derecho) y los métodos de enseñanza la dictatio y la disputatio; conformaron el currículo entendido como carrera o desplazamiento corto, que luego fue dividiéndose, ampliándose, adecuándose y especializándose con el paso del tiempo. 
El acomodo curricular de las 'artes liberales' tuvo entre otros fines introducir sistemáticamente al hombre en el universo de conocimientos elaborados por la humanidad a través de los siglos. En el currículo de las 'artes' y los procedimientos pedagógicos se buscaba dar nacimiento a un nuevo ser humano, engendrar y transformar al hombre de la ciencia y el saber.

Desde las épocas medievales, las universidades prestaron a la sociedad el ministerio de tres profesiones que respondian - empezaban a responder a las urgencias sociales de justicia, fe y culto, salud y educación. La "universitas" de la primera edad atendió a los anhelos de la humanidad, con las profesiones seculares, o por lo menos intentó hacerlo.

El paulatino desarrollo de las ciencias, de los sistemas económicos, sociales y culturales trajeron consigo el ingreso a la universidad de las profesiones modernas, con la consiguiente presencia de los currículos técnicos y profesionales en el sentido contemporáneo. Currículos de gran variedad, en función del incremento multiplicado de las diversas profesiones.

El concepto de profesión constituyó una atadura entre la 'universitas' y la sociedad de entonces. Después cambiaron las circunstancias sociales y políticas, y en uso de los avances logrados en el conocimiento, fue haciéndose mas acertado $y$ adecuado el servicio de la ciencia y de las profesiones al cuerpo social. A través de las profesiones la universidad prestó y sigue prestando un servicio a la comunidad.

En las profesiones lo mismo que en cada una de las diferentes especialidades, hay que distinguir la identidad particular de cada una, derivada de su objeto y fin propio como oficio concreto, y del cuerpo de ciencias que la sustentan. Las profesiones prácticas a diferencia de las académicas que profesan el servicio a la generalmente dichas ciencias "puras "- no son una ciencia. Dominantemente son artes, son quehaceres - las ciencias se diferencian entre sí por su grado o distancia de la aplicabilidad. De la aplicabilidad de cada ciencia surge la técnica, o conjunto de procedimientos y habilidades para realizar cosas sensorialmente perceptibles, al servicio de una necesidad o idea. La técnica denota la destreza, tanto para lo necesario y útil, como para lo bello y humano. Y este es el mundo de las artes, que las hay muchas; $y$, por lo tanto profesiones. Cuando un conjunto de ciencias, conjugando técnicas y artes que les son suyas, conforman un que hacer de aplicaciones prácticas y útiles, tenemos una nueva profesión; sin haber asistido por ello a la epifanía de una ciencia nueva. El haz de ciencias, son las ciencias de la profesión. Pero la profesión sigue siendo lo que es, una técnica, una arte útil y necesario para la sociedad. Lo que no quiere decir que las profesiones prácticas no sean profesiones cientificas. Si lo segundo no se diera- científicas fundamentadas en sus ciencias- se estaría frente a simples oficios ejercidos cual artesanos espontáneos y primerizos desinteresados del porqué científico de la labor realizada. Las ciencias sustentantes de una profesión y el objeto u oficio definen fundamentalmente la identidad de cada profesión. Las profesiones prácticas no son tanto ciencia cuanto artes; pero si hay ciencias de o para las profesiones, utilizables y conjugables curricularmente en gavilla interdisciplinaria, a efecto de poner sus técnicas al servicio del arte, que es la profesión. (Borrero, 1981)

\section{Docencia Universitaria Hoy}

La docencia es la actividad fundamental mediante la cual la universidad cumple su función de formar para el ejercicio de las artes, las ciencias, las profesiones y el desenvolvimiento personal y social. 
En la docencia confluyen en mutua interrelación las demás funciones de la universidad, pues al proceso de apropiación del conocimiento en el que participan activamente profesores y estudiantes, se integran los elementos de la investigación y en las acciones de interrelación prácticas en el estudio y solución de problemas de la realidad, se realiza la extensión.

El término Docencia proviene del latín 'Docentia' que significa ejercicio de la profesión docente, es decir el que enseña. La enseñanza es la intervención intencional, constitutiva de sentido, que realiza el profesor a través de la acción comunicativa e interacción humana, en el proceso de mediación entre la cultura, el conocimiento, y el estudiante, entre este, la misión de la universidad y el proyecto histórico social.

La cultura y el conocimiento, son el contenido para el desarrollo del intelecto, la personalidad, las aptitudes y los valores. Así la educación que acontece en la universidad como escenario de formación humana del más alto nivel, proporciona estructuras organizativas, currículos, medios de apoyo y personal dedicado a hacer esa formación, que exigen del quien enseña, una preparación especial.

El profesor como actor social, opta como profesión la misión de enseñar, labor compleja ya que en ella confluyen múltiples factores que son objeto de estudio de la ciencia pedagógica. La Pedagogía, ciencia sobre la enseñanza, ha desarrollado un campo intelectual de objetos, conceptos y métodos que trascienden la concepción instrumental, para ubicarla no solo como saber y como práctica sino como elemento integrador entre conocimiento, sociedad y cultura, y sus relaciones con los otros saberes, con la persona humana y sus formas de conocer. (Bernal, 2004).

Como resultado de la enseñanza, se da el aprendizaje del estudiante, actividad de apropiación de los conocimientos, de la producción científica, tecnológica y cultural. Se entiende por apropiación la adquisición y asimilación de conocimientos, habilidades y destrezas y la generación de actitudes que permiten la utilización, transformación o desarrollo de los objetos de apropiación. (U. Valle, 1981).

La enseñanza cumple dos funciones a saber: la instrucción y la formación. En la función de instruir la enseñanza juega un rol natural, siendo su responsabilidad esencial la de entregar de manera metódica y sistemática, completa y al día el conocimiento vigente de las disciplinas científicas y su aplicación en el ejercicio de las profesiones, según el campo que la docencia deba cubrir y esto, de un modo tal, que el estudiante pueda manejar y aplicar los elementos, estructuras y principios e incluso formularse y reformular las hipótesis, tesis y conclusiones a que le eleven su capacidad o su talento creador. En la función de formación, la enseñanza tiene también una participación destacada, solo que se realiza en un proceso más complejo, más sutil y más lento que en la instrucción. Pues, la educación entraña proveer una formación intelectual a la vez que ética, que conduzca al estudiante a examinar los conceptos, las formulaciones y las ideas, con una sano espíritu crítico, racional y objetivo; y a conformar una visión integrada de las ciencias, de modo que no se altere, por el contrario se afirme el delicado equilibrio que ha de mantenerse entre el avance de aquellas y el progreso moral y bienestar social de los hombres y sus comunidades.

Desde esta perspectiva, la universidad debe brindar una educación integral que enfoque a la persona del estudiante como una totalidad y no únicamente en su potencial cognoscitivo o en su capacidad para el quehacer técnico o profesional. El ámbito de la formación integral es el de una práctica educativa centrada en la persona humana y orientada a cualificar su 
socialización para que el estudiante pueda desarrollar su capacidad de servirse en forma autónoma del potencial de su espíritu en el marco de la sociedad en que vive y pueda comprometerse con sentido histórico en su transformación (Orozco, 1999).

En la formación humana integral, se busca en primer lugar, el desarrollo del talento, la creatividad, el discernimiento, la capacidad cognitiva, la sensibilidad, los valores y el desarrollo físico y espiritual de las personas, y en segundo lugar, preparar para su desenvolvimiento en las áreas del saber y las profesiones en los diferentes escenarios sociales

Los cambios de la sociedad, han ido generando distintos modelos de educación e integrando nuevos componentes al concepto de universidad y exigen desarrollar la capacidad de anticiparse y responder de manera adecuada a las necesidades e intereses de la sociedad para asegurar su pertinencia y su perdurabilidad.

Los desarrollos actuales de los procesos de globalización de la cultura y la economía, la democratización de los sistemas sociales, la expansión de los medios de información y accesibilidad al conocimiento como bien público, el avance de la ciencia, la técnica y los procesos interdisciplinarios en su producción, están generado el surgimiento de nuevas disciplinas especializadas y profesiones para responder a un mercado laboral complejo y competitivo. Cada vez más esta tomando auge el interés por la calidad en los procesos y en los productos de la educación, para lo cual se exige el dominio de competencias en los estudiantes y la generación de criterios o estándares de calidad, como mecanismos de certificación y acreditación que determinan el impacto social y la pertinencia de las universidades.

Las competencias se refieren al saber hacer en el contexto, es decir, al conjunto de acciones que un estudiante debe realizar en un ambiente

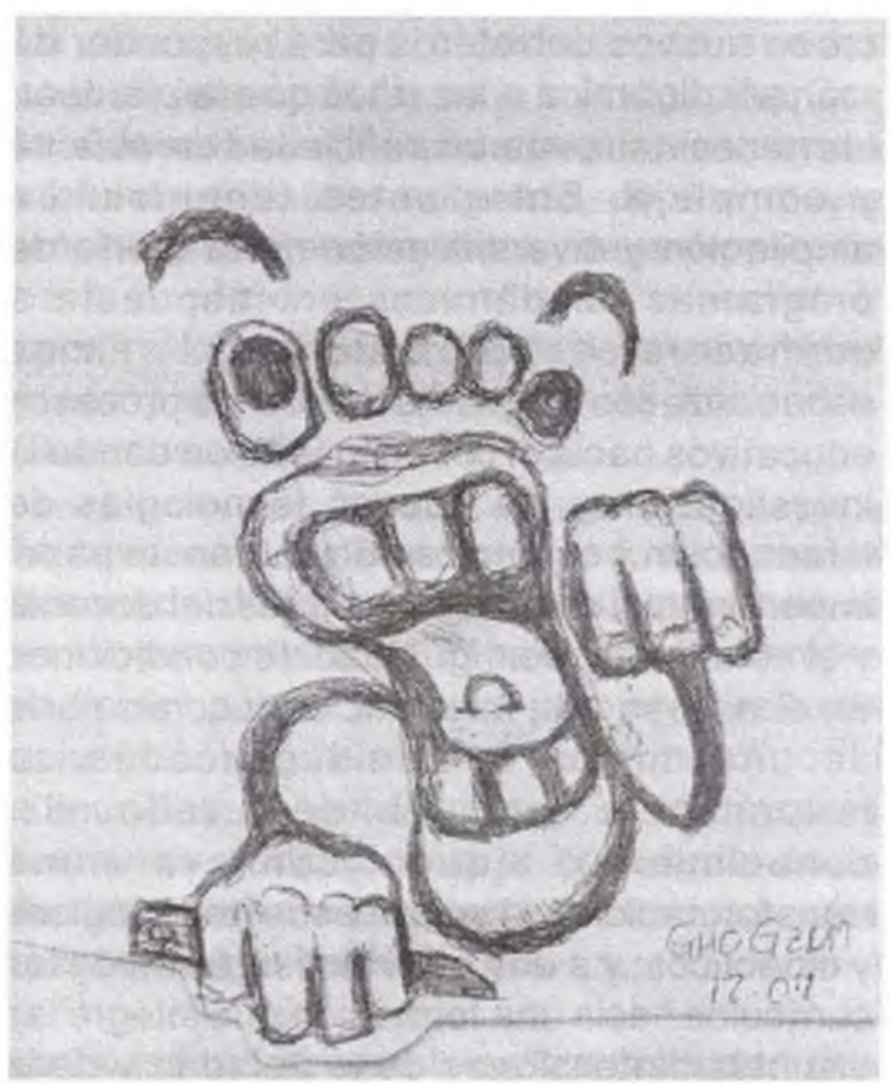

social en particular, para cumplir con las exigencias del mismo. Las competencias también son las capacidades de adaptación para el desempeño en campos nuevos. Las competencias determinan las metas educativas dirigidas a la formación del estudiante para que se desenvuelva adecuadamente en su medio social. Una persona competente combina efectivamente cuatro tipos de saberes: un saber conceptual (saber), un saber procedimental (saber hacer), un saber actitudinal (saber ser), y un saber meta cognitivo (saber aprender).

Los estándares, se conciben como los contenidos mínimos que debe saber un estudiante en un determinado nivel o campo de especialidad. Son guia referencial para que las instituciones ofrezcan la misma calidad de educación en un país y en relación con el mundo globalizado.

Estas nuevas exigencias están llevando a que las universidades adecuen la docencia y 
creen nuevos derroteros para responder de manera dinámica a las retos que le plantean las necesidades de una sociedad cambiante y compleja. Entre estos tenemos: La ampliación y diversificación de la oferta de programas académicos en respuesta a quehaceres profesionales mas especializados; la orientación de los procesos educativos hacia el conocimiento en donde la investigación y las nuevas tecnologías de información y comunicación cobran un papel importante; el cambio en los roles del docente y el estudiante, con igualdad de condiciones en el acceso a la información y como parte de una misma comunidad académica responsable por el desarrollo del conocimiento, que conlleva una transformación en los procesos pedagógicos y didácticos; y a una nueva orientación de los currículos hacia una formación que integre las distintas dimensiones de la persona y de la sociedad y brinde una mayor especialización en los campos de saber y en la formación profesional del estudiante. (Restrepo, 2004)

Se entiende por Currículo la organización que se hace del conocimiento, de los valores y habilidades, de los procesos pedagógicos mediadores entre los saberes y las personas, que permiten la formación humana, y de la disposición de los recursos tanto humanos como materiales necesarios en su desarrollo.

Por los cambios que deberán generarse en la docencia, los currículos deberá concebirse de una manera más flexible, interdisciplinaria, abierta e innovadora, estrechamente relacionado con los intereses sociales y económicos de la construcción de un nuevo proyecto nacional, más integral y, sobre todo, con contenidos vinculados a la producción y transferencia de conocimientos hacia la industria, la producción, la resolución de problemas sociales y naturales. Esta orientación concibe los procesos educativos desde una perspectiva que integra la formación humana, el desarrollo integral y los compromisos éticos del estudiante, con la capacitación para un ejercicio profesional adecuado y permanentemente cambiante. (Didriksson, 1995)

\section{La Investigación Universitaria}

El término investigación viene de 'investigiumire' o re-correr, re-pisar las huellas ya trazadas. Sin ser el único, este sentido de recoger y atesorar los hechos científicos de griegos y romanos, de árabes y hebreos fue el esfuerzo investigativo de la universitas, de indagarlo, comentarlo, difundirlo e integrarlo a la cultura. Tal, entre otros, el gran mérito de los primeros universitarios a partir del renacimiento cultural del siglo XII. (Borrero, 1986)

La investigación es la búsqueda sistemática de conocimientos nuevos que obedece a un proceso ordenado, programado y no al resultado de hechos al azar.

La investigación, tiene por objeto aprender, descifrar, interpretar, resolver, conocer y explicar los problemas, fenómenos y procesos de la realidad que se presentan como situaciones o estados del medio ambiente natural o social, del sistema o del propio sistema.

La investigación está encaminada a la solución de problemas. Su objetivo consiste en hallar respuestas a preguntas mediante el empleo de procedimientos científicos. Este proceso se inicia a partir de atributos propios del ser humano que lo han moldeado a lo largo de la historia. Se refiere al deseo de saber, de conocer el por qué, el cuando, el cómo de lo que sucede en la naturaleza y en la sociedad; también a la capacidad de cuestionarse, de buscar respuestas satisfactorias a los interrogantes; a ese deseo de investigar para comprender y poder utilizar la realidad en beneficio propio. Ese deseo por el conocimiento y la necesidad de investigar van aumentando en la medida en 
que se van logrando respuestas y por lo tanto planteándose nuevos interrogantes. (Guhl, 1986)

La investigación es parte de la explicación del desarrollo acelerado de la humanidad en todos los campos. Es por ello que la investigación científica es una de las bases principales de la civilización actual. De la investigación científica dependen la salud, el bienestar, la riqueza, el poder y hasta la independencia de las naciones. La investigación permite sobrevivir y progresar en medio de una competencia mundial en la que triunfan los paises que han dado empuje a los hombres de ciencia. (Houssay, s.f)

La Revolución científica condujo la investigación por los senderos de descubrimiento, del invento, del progreso de las ciencias naturales y sociales y su aplicación para beneficio de la sociedad.

Este mismo desarrollo, el perfeccionamiento de los métodos investigativos y de los instrumentos para hacerlo efectivo ha permitido en nuestros días la planificación de la investigación. Investigación planificada capaz de fijarse objetivos claros, no tan sometidos al azar; de presupuestar costos y determinar etapas, con la mayor certeza de llegar a cumplir con los propósitos determinados.

La investigación es el eje dinamizador de la vida universitaria, ya que ella permite la producción de conocimiento nuevo, el desarrollo de la ciencia y la tecnología, la solución de los problemas fundamentales de la sociedad y el mejoramiento de las condiciones de vida. La calidad académica de sus programas se garantiza a través de la investigación, puesto que sus resultados constituyen los contenidos del aprendizaje, y su vivencia permite la formación del espíritu científico, el desarrollo de la creatividad en los estudiantes, la vinculación con problemas de las ciencias y la orientación de las profesiones hacia las necesidades del medio.
La universidad es creadora de nuevos conocimientos mediante la investigación. Con la búsqueda científica de una compresión más profunda del saber adquirido, de una mayor ampliación de conocimientos y de su proyección hacia condiciones concretas de la sociedad, aporta al logro de los objetivos de la universidad y complementa la labor de la docencia. (Bateman, 1986)

La tendencia de la universidad, es pues, la búsqueda de la verdad. La búsqueda es la acción para el hallazgo. El hallazgo será siempre particular, parcial, limitado, pero el empeño es universal. La universidad se propuso como comunidad de varias generaciones de seres humanos dedicados a la búsqueda de la verdad, el intento de extender todos los bienes que derivan de sus hallazgos.

La investigación cumple en la universidad una función espiritual. El espíritu general es el de ser patrimonio de la universidad y a el debe servir la investigación, sin agotarse en el nivel simplemente utilitario e inmediato que ella persigue. El auge de la investigación puede deformar la misión universitaria si no hay una conciencia muy lúcida sobre su destino. Es necesario devolverle su carácter de educación esencial e integrante a las tareas investigadoras, porque si bien la investigación ha nacido para el crecimiento científico, ella no tendría sentido sino se forma a quien la desarrolla, la comunidad académica constituida por los docentes y los estudiantes.

El docente universitario no tiene por función única transmitir los conocimientos de su generación anterior a sus discipulos, sino que para él es absolutamente necesario aprender, buscar la verdad, ponerse a tono con los conocimientos de su generación sino del mundo en su día. Nadie ha definido si tiene que estudiar más y aprender más, el maestro o el estudiante. En ambos esa labor es cotidiana y esencial. Del mismo modo, cada miembro de la comunidad 
perteneciente a la generación joven no es simplemente objeto pasivo de la ciencia cumulada por la humanidad a lo largo de sus milenios. El universitario está dotado de bases que le permiten indagar verdades antes desconocidas por él y comunicarlas a los demás miembros del grupo mediante el diálogo y el trabajo en común. Esta función es a la vez discente y docente, ni más ni menos que la del profesor. De manera que la tarea de la universidad, aprender y transmitir, se cumple por todos sus miembros.

La universidad deriva su dinámica en la búsqueda de la verdad, del encuentro entre la tradición y novedad, o sea, de la erudición y la invención. La indagación metódica en un grupo de indagación de la verdad es la cátedra llámese laboratorio o seminario, dirigida siempre por lo más doctos y experimentados. Autoridad que en ninguna manera influye para que los conceptos sean tenidos como certeros sino en la medida de las pruebas que aporten se trata de que sean ellos quienes dirijan y orienten la búsqueda de la verdad por medio del método. No son siempre quienes enseñan, muy frecuente el miembro joven de la universidad es el que halla. Resulta así que no hay diferencia entre la docencia y la discencia, ni entre enseñar y aprender, ni entre enseñar, aprender e investigar. La investigación que es esta misma búsqueda de la verdad puede dividirse en erudición e invención.

Erudición, es el hallazgo de verdades ya conocidas por otros hombres pero que eran ignoradas por el miembro investigador de la universidad, docente o discente. Resulta una utopía que el universitario o al menos la comunidad universitaria posean todos los conocimientos ya descubiertos por la humanidad. Sin embargo, esa es la tendencia, ese el motivo y la razón de ser de la universidad, ello lo que dice su nombre. De ahí que no la definamos como poseedora de la verdad, ni siquiera como comunidad que encuentra la verdad, sino que la busca. La búsqueda es acción para el hallazgo. El hallazgo será siempre particular, parcial, limitado. Pero el conato es universal. Así son tan universidades las pequeñas sin grandes medios para el hallazgo frecuente como las numerosas y bien dotadas, siempre que estén empeñadas en esta dinámica de la búsqueda. La invención es hallazgo de verdades antes no conocidas por ningún hombre. No debe sin embargo pensarse en que haya diferencia fundamental entre la invención y la erudición. Solamente mediante la erudición y la aprehensión de los conocimientos tradicionales de la generación mayor, puede una generación dar este aporte maravilloso del invento, la mayor flor de la Universidad. Puede ser que en muchos lustros y aún siglos una comunidad universitaria determinada nada vistoso invente, y ello no le resta grandeza ni gloria, siempre que busque e indague. Pero no ha de creerse que el invento es solo la novedad tecnológica de aplicaciones útiles no observadas antes. La invención tiene campo vastísimo en las artes. La investigación creadora produce valores estéticos o la investigación histórica valores históricos o la disquisición metafísica planteamientos originales o el análisis sociológico hallazgo verdaderos.

Pese a lo anterior, mucho se discute hoy sobre la forma como la universidad debe asumir la investigación como función original y como función suya, aceptando, de entrada, que se trata de un deber y una actividad legítima y obligante. Algunas respuestas más frecuentes son: formar hombres pensantes, recursivos, de iniciativa, precisamente a través de la docencia unida a la investigación, no tan profesionalizados que respondan, a situaciones concretas y exigencias del trabajo, fortalecer los postgrados con carácter investigativo, para formar investigadores, y finalmente, acudir a la fórmula de la universidad-empresa investigativa que vende servicios al Estado y a la empresa, de manera especial. Compromiso, este último, 
que cumplirían unos cuantos, los investigadores de oficio, en los Institutos creados para tal fin. (Borrero, 1986)

\section{La Extensión Universitaria}

El significado de la palabra 'professio', en el léxico de la 'universitas' medieval, insinúa que las profesiones configuradas por entonces respondieron a necesidades sociales de la época: la salud, la justicia y la fe. Pero la historia no da indicios de que la 'universitas' hubiera tenido conciencia institucional del 'servicio' a la sociedad como hoy se entiende. Ni siquiera el nacimiento de las universitates ex - privilegio, muchas fundadas para suministrarle a los principados los servidores y administradores de la cosa pública, tuvieron conciencia institucional respecto a las funciones de servicio y extensión como hoy se entiende.

Debe aceptarse que a su modo, la 'universitas' estuvo presente en la vida de la ciudad. En las ciudades medievales en general muy pequeñas, la universidad ocupaba lugar importante. Ella confería prestigio a la ciudad y le hacia llegar medios económicos. Las construcciones, muchas veces financiadas por los reyes, daban ocupación a los artesanos y artistas. En ocasiones solemnes, en las fiestas de la ciudad y en las procesiones religiosas, los profesores y estudiantes, agrupados por facultades con sus estandartes y sus banderines ocupaban un lugar privilegiado. En periodos de enseñanza, en los semestres, los estudiantes se hacian presentes en todas partes, en las plazas y en las tabernas $y$, muchas veces, sus cantos y sus pendencias perturbaron la tranquilidad de los pacíficos vecinos. En cambio, en los periodos de vacaciones la ciudad parecía muerta. (Borrero, 1992).

La 'universitas' así hubiera estado en la ciudad, o mejor era la ciudad, no realizó empresas de extensión. Hubo acciones de la universidad con reacciones en los medios sociales, y también lo contrario, el tiempo que interacciones que pasan por los trechos de la historia hasta nuestros días. Por ejemplo, en el Renacimiento, la universidad y sus profesores solian intervenir en importantes asuntos públicos y en graves negocios de Estado, actividad acentuada en los agitados tiempos de la Reforma y de los descubrimientos para hacer frente a situaciones totalmente nuevas en que se plantearon difíciles problemas cuya solución requería erudición y sabiduría. Los grandes teólogos y juristas españoles de las universidades de Alcalá de Henares y de Salamanca, Juan de Santo Tomas, Francisco de Vitoria o Francisco Suárez, ejercieron una influencia decisiva sobre las resoluciones y la ley que acordó la Corona de España para dar forma al estado Indiano y definir las políticas que se debian seguir frente a las poblaciones indígenas en el mundo nuevo. Carlos $V$ asistió personalmente a las lecciones de Vitoria en Salamanca, y se inspiró en su escrito sobre los indios recientemente descubiertos y el derecho de los españoles de hacer la guerra a los bárbaros.

Lo anterior índica que pese a la presencia universitaria en la ciudad y al influjo ejercido en los asuntos de épocas sucesivas, no se manifestó la actividad de extensión o de servicio social tal y como se entiende hoy.

\section{Los Conceptos de Servicio y Extensión}

Según fuentes históricas, los conceptos de servicio y extensión en la universidad tuvieron su origen en las 'land-grant universities' de los Estados Unidos, dado que la sesión de tierras exigía como contrapartida universitaria desarrollar técnicas agrícolas y otras derivadas del estudio de las ciencias naturales. Otra fuente histórica se ubica en Inglaterra, en donde por siglos vio en las seculares Oxford y Cambridge sitial casi exclusivo de la educación superior; la expresión 'university extensión' se tradujo en 
que si era imposible que la masa social llegara a la universidad, no habría inconveniente en que las universidades llegaran a la masa.

Los sentidos dados a los designaciones de 'servicio' y 'extensión', permiten establecer diferencias en estos conceptos que a la vez pueden fundirse en la expresión comprensiva 'extensión del servicio'.

En el deslinde entre servicio y extensión, se ubican en el primero los servicios indirectos que la universidad presta a la sociedad y a la comunidad con la formación de profesionales, algunos más directos que derivan de la práctica de tal o cual modalidad operativa de la educación permanente no formal y los servicios de investigaciones especificas, a más de otras prestaciones de variada naturaleza.

Existen varias formas del servicio, la primera consiste en que la universidad facilite a la comunidad el uso de sus recursos específicos y de planta: laboratorios, bibliotecas, aulas y también sus recursos humanos de profesores, investigadores y estudiantes para acciones académicas, de campo, y varias otras. Segunda, que la universidad acepte solicitudes de la comunidad para estudiarle cuestiones y problemas especificos u ofrecerle concretos y convenidos programas de educación permanente. Que la universidad se sienta libre y ágil para seleccionar problemas que ella detecte en la comunidad, y los estudie sin solicitud previa del medio social. En fin, que la universidad se comprometa a ser ejecutora de las soluciones que plantea, ya se trate de estudios hechos a solicitud de la comunidad, o de aquellos que la universidad se propuso.

En la extensión, estarían las acciones que la universidad ejerce de la manera más abierta y general en beneficio de las comunidades y la sociedad, como son algunos modos operativos de la educación permanente y no formal que tienen como objeto la masa social y no los estudiantes regulares de la universidad. Dicho de otra forma, por extensión se entiende como los conocimientos que la universidad atesora y utiliza en beneficio de todos aquellos que nunca tuvieron contacto con la educación o que habiendo transitado por sus niveles, requieren poner al dia sus propios haberes intelectuales. La extensión corresponde a la educación permanente suplementaria, complementaria y continuada. Toda vez que cualquiera de estas modalidades operativas de la educación permanente constituye una forma de suplir, en el primer caso, y de extender en el presente situaciones inconclusas, en el caso de las restantes modalidades operativas. La extensión concebida como difusión cultural, corresponde a la educación permanente perfectiva y culturizante, en beneficio de unos y otros destinatarios.

\section{La Extensión Universitaria Hoy}

La función de extensión de la Universidad es el vínculo que ella establece con la comunidad para socializar el conocimiento que produce, aportar a la solución de problemas. enriquecer la cultura y orientar su destino presente y futuro. La extensión, tiene por objeto promover, divulgar, difundir, distribuir, suministrar y aplicar los conocimientos y servicios producidos. La extensión también es el medio de interacción social por el que la Universidad aprende de la realidad (saberes, sentidos, valores, símbolos, normas, roles...), evalúa los efectos de su acción y se construye a sí misma.

La primera extensión de la Universidad son sus egresados, a través ellos brinda a la comunidad el talento humano que ha de actuar en los diferentes escenarios sociales. Los egresados son la expresión y el aporte 
más significativo que la institución entrega a la sociedad.

La extensión también compete a los miembros de la comunidad universitaria. La parte compacta, el cuerpo de profesores y estudiantes, en sus tareas cotidianas, tienen la obligación de irradiar conocimientos y servicios sobre la comunidad.

La suprema función social de la universidad es ser laboratorio para la mejora del hombre, y en la práctica cada universidad para la mejora de su pueblo. Los miembros de la universidad que se consagran a la búsqueda de la verdad empiezan la carrera ocupando el último sitio de una comunidad parcial, pero la terminan recibiendo el sitio principal en la comunidad nacional. Es entonces cuando los hallazgos de la comunidad universitaria se vuelven infrenables sobre la comunidad nacional. Nada hay en las reformas sociales y en las revoluciones científicas que años antes no haya estado en la mente de los universitarios. La única manera de influir sobre la marcha del mundo o de la nación dentro de contados años, es influir hoy sobre la comunidad universitaria. (Henao, 1963).

Forman parte de la extensión las prácticas, los proyectos de aplicación y transferencia de tecnologías, las actividades de servicios a la comunidad como las acciones de educación no formal (diplomados, cursos, seminarios, talleres, encuentros, conferencias, foros $y$ demás eventos que se hacen sobre temas de interés general o específico para ciertos sectores o poblaciones); las de educación informal (muestras, exposiciones $y$ exhibiciones de diferentes productos de la academia; publicaciones como boletines, periódicos, revistas libros, programas de televisión y radio; asesorías, consultorías y la asistencia especializada para asuntos técnicos puntuales; los servicios de laboratorio y los proyectos de desarrollo comunitario.

\section{Las Urgencias Universitarias}

Plenamente conformados los Estados modernos, las funciones clásicas de la Universidad en orden al hombre (Docencia), a la ciencia (Investigación) y la sociedad (Servicio o Extensión), en el siglo XIX empezaron a entretejerse con la triada de las hoy llamadas urgencias universitarias.

La terminologia misma de 'urgencias universitarias' es contemporánea. Pero sus prenuncios, datan del siglo XIX.

El término, introducido por el filósofo francés Paul Ricoeur, denota la reactiva conciencia institucional que plantea acuciantes inquietudes respecto al ejercicio de las funciones de la universidad e indirectamente el carácter manifiesto de sus notas. Ricoeur, define tres urgencias o instancias raciones que caracterizan la conciencia universitaria: La urgencia crítica, la urgencia política y la urgencia cultural.

La urgencia o conciencia critica, racional por tanto, es aquella mediante la cual la universidad juzga los efectos de la educación y la ciencia sobre el hombre, la sociedad, la nación y el mundo, porque la universidad es universal.

La urgencia política, responde a posturas nacionales desde el desarrollo y el estudio honesto de las ciencias, con la actitud responsable del individuo ante sus deberes ciudadanos y con la búsqueda de mejores acuerdos del poder del saber y del poder político, hacia un humano concepto del desarrollo social que esté revestido del nombre de la paz. Con todo, en no pocas ocasiones la opción política significa entrega de la universidad como tal a la acción electorera y partidista, cuando la institución acepta someterse a conducciones exógenas e interesadas. También se presta esta 
conciencia, absurdamente interpretada, a la violencia y al atropello.

La urgencia cultural. en su cometido de promoción del hombre integral, la universidad presta un servicio a la toma de conciencia y a la profundización de la identidad cultural, proceso dinámico en el cual se recrea en el presente el patrimonio del pasado y se proyecta el futuro, para que sea asimilado por las nuevas generaciones y de este modo asegurar la identidad y el progreso de un grupo social.

La educación es una actividad humana en el orden de la cultura, no sólo por ser la primera y esencial tarea de ésta, sino también porque la educación juega un papel activo, crítico y enriquecedor de la cultura misma. La universidad es un campo propicio, para orientar eficazmente la cultura de una nación. Debe servir al país en el esfuerzo común por construir una sociedad nueva, libre, responsable, consciente de su propio patrimonio cultural, justa, fraterna, participativa, donde el hombre integralmente considerado, sea siempre la medida del progreso.

Como centro de ciencia, la universidad es cosmopolita porque la ciencia es internacional y no local. Pero como órgano cultural su función es más restringida porque la cultura si tiene límites sociales y geográficos. Frente a los problemas nuevos de la universidad esta forzada a prevenirlos y dilucidarlos, pero a lo que no le es dado es a resolverlos porque entonces sobrarían los gobiernos. Su objetividad debe ser tan evidente, que los gobiernos puedan tener sus soluciones, como una dé las alternativas que se le den entre muchas otras instituciones ocupadas de los mismos problemas.

\section{Las Urgencias en el Desarrollo de la Universidad.}

La educación, hecho individual pero también social, ha sido inseparable de la cosa política. Así lo entendió la antigüedad hasta llegar a ser pensamiento de Platón y de Aristóteles.

Nacidas las universidades como instituciones sociales para la ciencia y lo superior educativo su identidad fue parte del fenómeno urbanístico y político de la Edad Media. Los poderes civiles medievales sintieron que las universidades podian serles útiles y nacieron las 'universidades 'exprivilegio' fundacional.

En el renacimiento, cuando empezaron a gestarse los estados modernos, la universidad menos fiel a su nota originaria de universalidad se vistió de ribetes nacionales.

La nota científica de la universidad entró en juego. Desde el siglo XIX se vislumbró la contienda en torno a la neutralidad de la ciencia, en contraste de la ciencia comprometida con los intereses sociales, políticos y nacionalistas. Nació muy pronto la cuestión de si podría dar una ciencia objetiva, desasida de acuerdos. Un siglo después, Max Weber se convertiría en decidido luchador por una ciencia libre de valores, porque habia recogido y solucionado el debate entre ciencia y política nacionalista. Pero la ciencia disgregada sucumbió a la politización y con ella la universidad.

Más tarde el desarrollo industrial, las implicaciones políticas y económicas sobre la universidad; el positivismo científico, la dispersión del saber, y los nuevos acercamientos de la técnica a los dominios de la ciencia, contribuyeron a configurar la tecnología y a convertirla en factor social y en motivo más consciente de la educación.

No es de extrañar entonces que apenas iniciado el siglo, vieran la luz primera los tecnológicos al igual que la universidad y se anunciaran las primeras desmembraciones del nivel postsecundario de la educación. 
Se fortalecieron entonces las universidades y los 'colleges' técnicos y, en nivel más sencillo, las instituciones mecánicas o de artes y oficios. De tal manera que el entrenamiento para las profesiones nuevas y la capacitación universitaria para la industria y el comercio, llegaron a ser funciones e hilo conductor del desarrollo científico de la educación superior. Aparecieron los sistemas postsecundarios, algunas universidades hicieron suya tan obligante función, en algunos paises muchas instituciones especiales fueron creadas para atender a la emergente necesidad. (Borrero, 1992)

El siglo XIX también ensayó los conceptos de desarrollo social, desde entonces tan cercanos a lo económico. De desarrollo planificado -la planeación-, que en sus sistematizados proyectos envolvió el mundo universitario. Apareció la universidad lanzada hacia propósitos cada vez más específicos e intencionados.

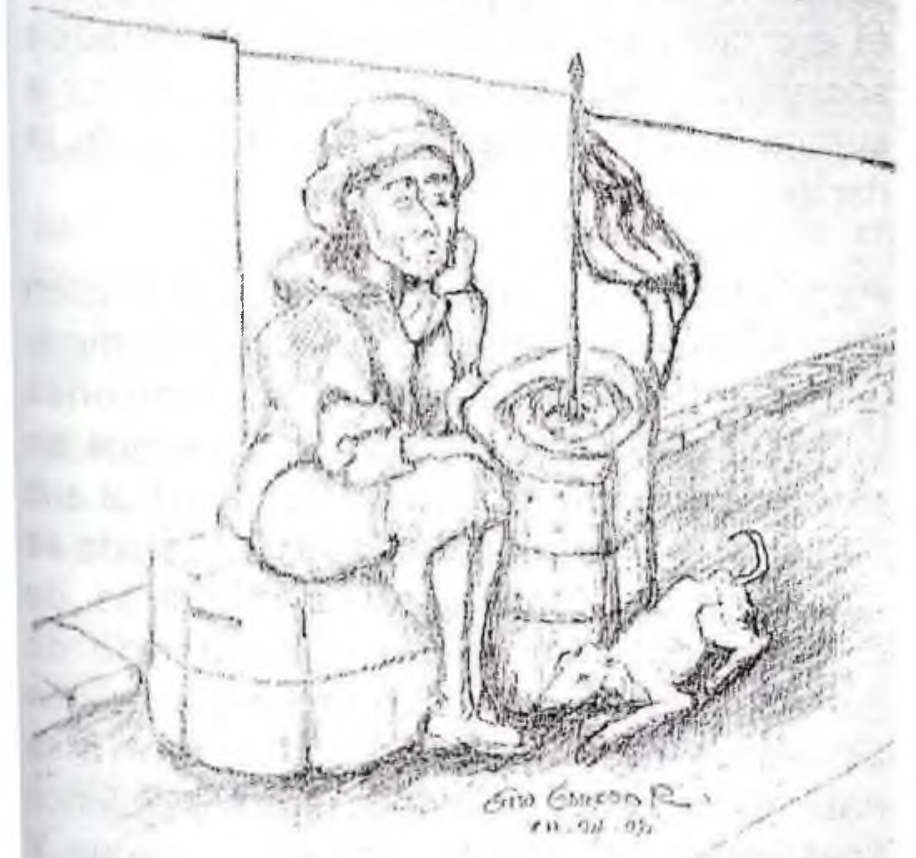

A partir de este momento la planeación educativa unió, sus destino a las proyecciones económicas, toda vez que la educación empezó a concebirse como importante elemento del progreso material y económico de las naciones. Cundió el convencimiento de que sin educación de todos no podría alcanzarse la estabilidad política.

Así, todos los Estados modernos se penetraron de su responsabilidad en la promoción educativa. Los currículos, por supuesto fueron objeto de interés y también de intervención. También la formación de los maestros y estudiantes.

La Universidad de antaño que hacía de sus egresados maestros y doctores se vio obligada a brindarle alojamiento a las nuevas profesiones técnicas, tecnológicas y sociales ansiosas de alcurnia superior.

Ante las situaciones nuevas establecidas, fruto de las condiciones políticas y las configuraciones nacionales, surgió la urgencia de la nueva función crítica que en nuestro siglo se extiende a los el análisis de la relaciones con los Estados, la política y el ejercicio de la ciencia.

Las funciones universitarias tuvieron una insistencia o sesgo de tipo individual o particular. Ya sea que la función ejercida haya tenido como término a la persona o a cada una de las ciencias o sectores del conocimiento en particular. Pero la universidad tuvo y tiene funciones sociales. Por ello, se habla del servicio a una sociedad dada, de su desarrollo o de su cambio. Aquí el término de la acción no es el individuo solamente, ni una ciencia particular, sino el complejo político de los individuos unidos en sociedad. La formación de profesionales, es algo que se llevó a cabo en cada individuo pero para el servicio de los demás. $Y$ también se ocupa hoy la universidad de la forma y modos de servicio que la ciencia ha de prestar a la sociedad humana. 
Modernamente esto se denomina la política de la ciencia. La función docente o educativa del hombre, se conjugan en otra que llamamos función magíster dota individual. Es la cultura personal que en buena parte perece con la muerte del poseedor. Las funciones sociales de la universidad- las profesiones sobreviven a la desaparición sucesiva de los profesionales- y el efecto político de la ciencia, constituyen rasgos de otra cara de la cultura que es la cultura social.

En consecuencia se asigna a la universidad otra función, la gestión de la cultura. También es necesario recordar que el hombre y la sociedad aspiran siempre a los mayores aciertos en la elaboración de la ciencia por la investigación justa de las relaciones sociales; en los verdaderos valores de las culturas individuales y de la cultura social, y en la configuración de una patria grande y bienhechora. Por tanto, se aceptó siempre - y hoy aún más- que la universidad ha de tener una función o actitud permanente crítica del valor de los conocimientos y sus métodos de investigación, sobre los efectos docentes, educativos, profesionales y culturales producidos en la persona y sobre el modo de sociedad que se está propiciando como resultado de la acción científica universitaria" (ASCUN, 1983)

La búsqueda incondicional de una verdad sin restricciones es la naturaleza de la universidad. La búsqueda de la verdad por los diversos caminos de todas las ciencias y de todos los saberes tiene que ser crítica y estar acompañada de la actitud reflexiva, dialogal y deliberante, con respecto no sólo con lo que procede del contexto sino con lo que ella hace o produce, sus incidencias en el sentido auténtico de lo ético y político de la pertinencia en función de las necesidades sentidas y demostradas. La indagación misma de los requerimientos y su análisis científico, unida a la necesidad institucional de proyectarse sobre el medio y aún de influir sobre las orientaciones del Estado como dinámica para el desarrollo actitud eminentemente política de la universidad, es propiamente la actuación social más inmediata y directa, pero además el principal deber como institución racional. La universidad debe aceptar plenamente esa responsabilidad, de cuya satisfacción no sólo depende su propia realización institucional, sino el prestigio social y la verdadera necesidad de ella que se logre formar el pais.

El logos de la universidad índica una especie de ambivalencia institucional al distinguir entre universidad como servicio público que se incrusta dentro de la educación que da lugar al correspondiente jurídico y la universidad como voluntad de saber socializado que origina la institución cultural. Esta distinción entre la doble imagen de la universidad al establecer sus nexos naturales y sus vínculos convencionales con el Estado, son materia de regulación y aluden a la existencia y función de la universidad. En este sentido, la universidad sostiene relaciones jurídico - legales con el Estado, en cuanto provee del servicio de la educación superior. La Universidad es corporación educativa de docentes y estudiantes, forma para el ejercicio en las diferentes actividades sociales, recoge, conserva y crea el saber y la cultura, es organización fundamental de la nación.

Para describir la relación que pueda existen entre Universidad y Estado, hay que determinar la naturaleza de cada uno, las funciones constitucionales de sus comportamientos en razón de su capacidad y de sus fines. Por ello hablar de sobre la Universidad y el Estado es asunto de disciplinas sociales y jurídicas, de criterios políticos y culturales dentro de contextos ideológicos. Sin embargo, existen comunes denominadores. En teoría la naturaleza política del estado determina ciertos fines entre los que se destaca el bien común. $Y$ la naturaleza de la Universidad también política en cuánto institución educativa y como institución 


\section{PanEasurcolombiana 17}

cientifica y cultural indica sus objetivos sobre el bien común. (Medellín, 1980)

Si bien la universidad vino a ser concebida como fuerza inspiradora del Estado, del desarrollo cultural y social, como institución receptora, conservadora y creadora del saber, responsable de él y de su influencia sobre la cultura, no puede rebajarse ni ser instrumento ciego de Estados, partidos políticos, clases sociales o grupos, sea cual fuere su orientación. No es la política la que debe dictar el rumbo a la universidad, sino que es ella misma quien debe interpretar las posibilidades más profundas de un pueblo. Ni la universidad, ni la sociedad tienen sentido si no es en función del bienestar humano. (Latorre, 1978).

Por su naturaleza como institución social y por los objetivos de su responsabilidad, la universidad es ente político. La concepción de la universidad en su significado político ha de servirnos para una aproximación de la universidad y el Estado.

\section{Bibliografía}

ASOCIACIÓN

COLOMBIANA

DE UNIVERSIDADES-ASCUN (1983). Autoevaluación institucional universitaria. El Nivel académico. Simposio permanente sobre la universidad. Segundo seminario general 1.983 - 1.985. Bogotá,

BATEMAN, Jaime. (1986). La Investigación en Ingeniería. Quinta reunión de Decanos de Ingeniería. ICFES. Serie Memorias de Eventos Científicos Colombianos. No.39. Bogotá.

BERNAL DE ROJAS, Aura Elena. (2004) Educación, pedagogía y modelos pedagógicos. Universidad Surcolombiana. Neiva.

BORRERO, Alfonso (1981). La identidad de las profesiones modernas en la universidad, el oficio y el trabajo. Revista mundo universitario. No.16. Bogotá.

BORRERO, Alfonso (1986). Conceptos universitarios en su desarrollo histórico. Reflexiones Universitarias: Universidad, Historia e
Investigación. Publicaciones Universidad Central. Bogotá.

BORRERO, Alfonso (1992). La Universidad desde 1800 hasta 1.945. Los Modelos Universitarios. Simposio Permanente sobre la Universidad. Quinto seminario General 1.990- 1.992. ASCUN- FESICFES. Bogotá.

BORRERO, Alfonso (1992). La Educación permanente o no formal y la función universitaria de extensión. Simposio Permanente Sobre la Universidad. Quinto seminario General 1.9901.992. FES-INCFES. Bogotá,

DIDRIKSSON, Axel. (1995) El futuro de la educación superior en Latinoamérica y el Caribe. Memorias del seminario UNAM/UNESCO. México.

GULH M, Ernesto. (1986) La investigación en Colombia. Dependencia y desarrollo propio. Quinta reunión de Decanos de Ingenieria. ICFES. Serie memorias de eventos científicos colombianos. No.39. Bogotá.

HENAO, Felix. (1963) Función social de la universidad y modo de ejercerla. Revista Bolivariana. No.93. Medellín.

HOSSAY, Bernardo (SF). La investigación Científica. Buenos Aires. Editorial Colombiana. MEDELLÍN, Carlos (1980). Universidad y Estado. Unión de Universidades de América Latina. VIII Asamblea General, 11 -14 de noviembre de 1.979. Universidad Autónoma de México.

LATORRE, Eduardo (1978). Algunas Reflexiones sobre la Misión y Organización de la Universidad Moderna. Asociación de Universidades e Institutos Investigación del Caribe. Isla Margarita (Venezuela).

OROZCO, Luis Enrique (1999). La Formación Integral. Mito y Realidad. Ediciones Uniandes. Bogotá.

RESTREPO, José María y LOCANO, Fernando (2004). Reflexiones sobre el Sistema de Créditos Académicos. Memorias Encuentro Nacional de Vicerrectores Académicos. Universidad del Rosario. Bogotá.

UNIVERSIDAD DEL VALLE (198). Programa de Magister en Docencia Universitaria. Cali. 